\title{
Prospects and limitations of expansion microscopy in chromatin ultrastructure determination
}

\author{
Ivona Kubalová • Markéta Schmidt Černohorská • \\ Martina Huranová • Klaus Weisshart • \\ Andreas Houben • Veit Schubert (1)
}

Received: 1 June 2020 /Revised: 28 July 2020 / Accepted: 5 August 2020 / Published online: 17 September 2020

(C) The Author(s) 2020

\begin{abstract}
Expansion microscopy (ExM) is a method to magnify physically a specimen with preserved ultrastructure. It has the potential to explore structural features beyond the diffraction limit of light. The procedure has been successfully used for different animal species, from isolated macromolecular complexes through cells to tissue slices. Expansion of plant-derived samples is still at the beginning, and little is known, whether the chromatin ultrastructure becomes altered by physical expansion. In this study, we expanded isolated barley nuclei and compared whether ExM can provide a structural view of chromatin comparable with superresolution microscopy. Different fixation and denaturation/digestion conditions were tested to maintain the chromatin ultrastructure. We achieved up to 4.2-times physically expanded nuclei corresponding
\end{abstract}

Responsible Editor: Jiming Jiang

Electronic supplementary material The online version of this article (https://doi.org/10.1007/s10577-020-09637-y) contains supplementary material, which is available to authorized users.

I. Kubalová · A. Houben • V. Schubert $(\bowtie)$

Leibniz Institute of Plant Genetics and Crop Plant Research (IPK)

Gatersleben, 06466 Seeland, Germany

e-mail: schubertv@ipk-gatersleben.de

M. Schmidt Černohorská · M. Huranová

Laboratory of Adaptive Immunity, Institute of Molecular

Genetics,, Academy of Sciences of the Czech Republic, Prague,

Czech Republic

K. Weisshart

Carl Zeiss Microscopy GmbH, 07745 Jena, Germany to a maximal resolution of $\sim 50-60 \mathrm{~nm}$ when imaged by wild-field (WF) microscopy. By applying structured illumination microscopy (SIM, super-resolution) doubling the WF resolution, the chromatin structures were observed at a resolution of $\sim 25-35 \mathrm{~nm}$. WF microscopy showed a preserved nucleus shape and nucleoli. Moreover, we were able to detect chromatin domains, invisible in unexpanded nuclei. However, by applying SIM, we observed that the preservation of the chromatin ultrastructure after the expansion was not complete and that the majority of the tested conditions failed to keep the ultrastructure. Nevertheless, using expanded nuclei, we localized successfully centromere repeats by fluorescence in situ hybridization (FISH) and the centromerespecific histone H3 variant CENH3 by indirect immunolabelling. However, although these repeats and proteins were localized at the correct position within the nuclei (indicating a Rabl orientation), their ultrastructural arrangement was impaired.

Keywords Chromatin - Expansion microscopy · Nucleus $\cdot$ Structured illumination microscopy $\cdot$ Hordeum vulgare

\section{Introduction}

Expansion microscopy (ExM) is a method to enlarge small structures physically in an isotropic manner to overcome the diffraction limit of light microscopy. Thus, super-resolution $(<250 \mathrm{~nm})$ can be realized cost-efficiently with diffraction-limited light 
microscopes (Chen et al. 2015; Chang et al. 2017). Even a lateral resolution of $\sim 70 \mathrm{~nm}$ can be achieved by combining ExM and standard confocal microscopy (Jiang et al. 2018).

ExM is based on a swellable polyelectrolyte gel, increasing in size when exposed to water to achieve a $~ 4.5$-fold three-dimensional (3D) expansion (Alon et al. 2019; Wassie et al. 2019). The first ExM protocol, expanding mouse brain tissue 4.5-times, was described by Chen et al. (2015). Since then, several expansion protocols emerged to increase the expansion factor and to preserve the ultrastructural features. These protocols were adapted to species like fungi, human, mouse, fruit fly and zebrafish and soft tissues such as brain, skin, kidney and liver (Chen et al. 2015; Tillberg et al., 2016; Cahoon et al. 2017; Freifeld et al. 2017; Halpern et al. 2017; Jiang et al. 2018, Lim et al. 2019; Truckenbrodt et al., 2019; Götz et al. 2020; Zwettler et al. 2020b). The following processes occur during ExM to fix, embed and expand the specimen successfully: (1) during the fixation with a formaldehyde/acrylamide mixture, formaldehyde crosslinks proteins/DNA/RNA to each other; (2) during gelation, the crosslinked proteins become crosslinked to the polyacrylamide (PAA) gel due to the acrylamide provided during fixation; (3) during denaturation in SDS buffer and at high temperature, all crosslinked proteins denature while remaining crosslinked to the PAA gel mesh which starts to expand in the denaturation buffer; (4) during expansion in water, all proteins renature back with gaps between each other but still bound to the PAA gel mesh preserving their exact position as before expansion (Chen et al. 2015; Cho et al. 2018; Tillberg and Chen 2019; Wassie et al. 2019).

At the subcellular level, expansion and superresolution microscopy have been combined to analyse fruit fly, mouse synaptonemal complex protein components and centrioles (Cahoon et al. 2017; Wang et al. 2018b; Xu et al. 2019; Zwettler et al. 2020a). Superresolution microscopy techniques such as structured illumination microscopy (SIM) are subdiffraction imaging methods bridging the resolution gap between light and electron microscopy. They were applied successfully in cell biology (Fornasiero et al. 2015) at specimens from both prokaryotes and eukaryotes and allowed also discovering new structures within plant chromatin (Schubert 2017). The multiplication of the achieved physical and optical resolution of both methods could also be useful to decipher the 3D structure of chromatin in cell nuclei and highly condensed metaphase chromosomes.

ExM was successfully applied to visualize specific proteins and RNAs by immunolabelling and in situ hybridization, respectively (Chen et al. 2016; Chozinski et al. 2016; Asano et al. 2018). Labelling of specific DNA sequences in spatially expanded chromatin has not yet been reported. Only the application of DNA-specific dye like DAPI was shown in combination with ExM (Zhao et al. 2017; Düring et al. 2019).

Physically expanded nuclei and chromosomes in combination with optical super-resolution microscopy to increase the resolution would allow analysing the ultrastructure, dynamics and function of chromatin more in detail, especially via the detection of DNA sequences and proteins after specific fluorescence labelling. Until now, the preservation of the ultrastructure of expanded chromatin has not yet been analysed by super-resolution microscopy. A previous study showed that isolated barley chromosomes can become expanded after gentle fixation and flow-sorting (Endo et al. 2014). However, whether the chromatin ultrastructure of these expanded chromosomes is preserved has not been analysed.

Caused by varying refractive indices of plant cell organelles, which induce spherical aberrations and light scattering (Komis et al. 2015), plant cell imaging is more challenging than imaging of prokaryotic and animal/human tissues. Due to the absence of cytoplasm, isolated and flow-sorted nuclei are well suitable to perform immunolabelling and FISH followed by SIM (Schubert and Weisshart 2015; Weisshart et al. 2016; Schubert 2017).

To test whether expansion microscopy could be applied to improve the ultrastructural analysis of somatic plant chromatin, we isolated interphase nuclei of barley and tested different preparation methods based on an advanced ExM protocol for ultrastructures, called ultrastructure expansion microscopy (U-ExM) (Gambarotto et al. 2019). We achieved a physical $\sim 4$.2-fold nuclei expansion and the partial preservation of the chromatin ultrastructure as proven by standard wide-field microscopy. Besides, ExM was combined with immunolabelling and FISH to analyse the interphase centromeres of barley. However, after examining the expanded chromatin by super-resolution microscopy, we noticed that the chromatin substructure was altered due to ExM. 


\section{Materials and methods}

Plant material and nuclei isolation

The root tips of barley (Hordeum vulgare L. var. "Morex") seedlings were collected in a fixation solution (formaldehyde (FA), glutaraldehyde (GA) or glyoxal) mixed with acryl amide and treated 5 min under vacuum, followed by incubation on ice for the indicated time (Table 1). After fixation, at least 100 root tips were washed twice with a $1 \times$ PBS solution and immediately chopped using a razor blade in $400 \mu \mathrm{l}$ nuclei-isolation buffer LB01 (Doležel et al. 2007). The nuclei suspension was filtered using a $50 \mu \mathrm{m}$ filter mesh (CellTrics ${ }^{\circledR}$, SYSMEX), collected into a new tube and stained with 4',6-diamidino-2-phenylindole (DAPI) $(\sim 5 \mu \mathrm{g} / \mathrm{ml}$, Molecular Probes no. D1306). Round coverslips (Ø12 mm) (Thermo Scientific, Menzel Gläser) were placed into a 24-well culture plate (Greiner) and coated with poly-Llysine for at least $20 \mathrm{~min}$. The dispensable poly-L-lysine solution was removed, and the prepared coverslips were used immediately or stored at room temperature (RT) in wells for later use. To load a coverslip with nuclei, the nuclei suspension was pipetted into the well containing a coverslip and centrifuged at $1000 \times g$ for $10 \mathrm{~min}$ at RT using a swing-bucket rotor (Eppendorf Centrifuge 5810 $\mathrm{R})$. The supernatant can be re-used for additional coverslips.

Gelation, denaturation/digestion and expansion

Before gelation, the nuclei-loaded coverslip was taken out from the 24-well culture well without drying. Per gel, $50 \mu$ monomer solution (MS) (Suppl. Table 1) was mixed with tetramethylethylenediamine (TEMED) and ammonium persulfate (APS) (final concentration $0.2 \%$ $\mathrm{w} / \mathrm{w})$, and $35 \mu \mathrm{l}$ of the resulting mixture was dropped onto the clean surface of an ice-cold plastic plate covered by parafilm. The nuclei-loaded coverslip was promptly placed on top of the gel drop with nuclei facing the gel. The plate was kept on ice for $5 \mathrm{~min}$ to allow the gel to solidify. To finalize the solidification process, the gel was placed into a wet chamber and incubated for $15 \mathrm{~min}$ at $37^{\circ} \mathrm{C}$. The solidified gel was carefully removed from the coverslip using flat forceps and submerged into either a denaturation or digestion buffer (Suppl. Table 1) or water and incubated as designed in Table 1. The simplified schema of the protocol steps are shown for monomer solution 1 and 2 in Fig. 1 a.
In the case of a digestion step, before the incubation with a digestion buffer, proteinase $\mathrm{K}$ was added at a final concentration of $8 \mathrm{U} / \mathrm{ml}$. After denaturation/digestion, the gel was expanded in distilled water until the expansion reached the maximum possible size of $\sim 50.4 \mathrm{~mm}$ ( $\sim$ 4.2 times expansion). The distilled water was changed at least three times. Due to the presence of the nuclei population from distinct cell cycle stages and technical impossibility to visualize nuclei in the gel before expansion, the expansion factor was estimated only on the gel expansion, from 12 to $50.4 \mathrm{~mm}$.

Non-denaturating (ND) FISH

A nuclei-containing fragment of the expanded gel was incubated in $50 \%$ deionized formamide in $2 \times \mathrm{SSC}$ for $20 \mathrm{~min}$ at RT. Afterwards, formamide was replaced by $50 \mu \mathrm{l}$ of hybridization mixture containing $50 \%$ deionized formamide, $2 \times$ SSC and 200 pmol of the $5^{\prime}$ FAMlabelled barley centromere-specific oligo probe $(\mathrm{GA})_{15}$ (Zhang et al. 2019). The gel was incubated in a sealed chamber for $22 \mathrm{~h}$ at $37^{\circ} \mathrm{C}$. The exposure to the hybridization solution containing ions (hypertonic) results in shrinkage of the expanded gel. Therefore, after hybridization, the remaining hybridization mixture was removed, and the gel was re-expanded in distilled water followed by microscopical observation. The simplified schema of these protocol steps is shown in Fig. $1 \mathrm{~b}$.

Indirect immunolabelling

A nuclei-containing fragment of the expanded gel was incubated with primary rabbit antibodies against the centromeric $\mathrm{H} 3$ variant $\mathrm{CENH} 3$ of barley (Houben et al. 2007) diluted 1:1000 in $400 \mu$ l of antibody solution $(2.5 \%$ BSA, $0.05 \%$ Triton X-100, $1 \times$ PBS) in a 12 well culture plate (Greiner) and incubated overnight for at least $15 \mathrm{~h}$ at RT. Next, the primary antibody solution was removed, and the gel was re-expanded in distilled water until it reached the size before antibody incubation. Afterwards, the gel was incubated with secondary anti-rabbit Alexa488 antibodies (1:200, no. 711-545152, Jackson ImmunoResearch) in $400 \mu$ l antibody solution and incubated at $37^{\circ} \mathrm{C}$ for $1 \mathrm{~h}$ followed by $2 \mathrm{~h}$ at RT. Additionally, 3-5 $\mu \mathrm{g} / \mathrm{ml}$ DAPI solution can be added together with the secondary antibodies to enable chromatin staining. Finally, the gel was re-expanded in distilled water and subjected to microscopic observation in a $22 \times 22-\mathrm{mm}$ coverslip chamber "Chamlide" (Live 
Table 1 ExM protocol variants for isolated barley nuclei using monomer solutions 1 and 2

Monomer solution 1 (MS1)

\begin{tabular}{|c|c|c|c|c|c|}
\hline $\begin{array}{l}\text { Protocol } \\
\text { number }\end{array}$ & Fixative & Time of fixation & Denaturation & $\begin{array}{l}\text { Expan- } \\
\text { sion }\end{array}$ & $\begin{array}{c}\text { Chromatin } \\
\text { structure }\end{array}$ \\
\hline $1 \mathrm{~A}$ & $\begin{array}{l}2 \% F A+2 \% \\
A A\end{array}$ & $12^{\prime}$ & $\begin{array}{l}\text { denaturation in SDS } \\
\text { buffer at } 96^{\circ} \mathrm{C}, 5^{\prime}\end{array}$ & ++++ & + \\
\hline $\begin{array}{l}\text { 2A (see suppl. } \\
\text { fig. 1) }\end{array}$ & $\begin{array}{l}2 \% F A+2 \% \\
A A\end{array}$ & $12^{\prime}$ & $\begin{array}{l}\text { denaturation in SDS } \\
\text { buffer at } 80^{\circ} \mathrm{C}, 3^{\prime}\end{array}$ & ++++ & + \\
\hline $\begin{array}{l}\text { 3A (see suppl. } \\
\text { fig. 3) }\end{array}$ & $\begin{array}{l}2 \% \mathrm{FA}+2 \% \\
\mathrm{AA}\end{array}$ & $12^{\prime}$ & $\begin{array}{l}0.2 \mathrm{M} \mathrm{NaOH} \text { in } 70 \% \\
\text { EtOH, RT, } 3^{\prime}\end{array}$ & ++++ & +++ \\
\hline $4 \mathrm{~A}$ & $\begin{array}{l}2 \% \mathrm{FA}+2 \% \\
\mathrm{AA}\end{array}$ & $12^{\prime}$ & no denaturation & ++++ & ++ \\
\hline $5 A$ & $\begin{array}{l}2 \% \mathrm{FA}+2 \% \\
\mathrm{AA}\end{array}$ & $\begin{array}{l}\text { 1h RT } \rightarrow>\text { overnight } \\
\text { at } 4^{\circ} \mathrm{C}\end{array}$ & no denaturation & + & + \\
\hline $6 \mathrm{~A}$ & $\begin{array}{l}1 \% \mathrm{FA}+1 \% \\
\mathrm{AA}\end{array}$ & $12^{\prime}$ & no denaturation & ++++ & ++ \\
\hline $7 A$ & $\begin{array}{l}1 \% \mathrm{FA}+1 \% \\
\mathrm{AA}\end{array}$ & $20^{\prime}$ & $\begin{array}{l}\text { denaturation in SDS } \\
\text { buffer only, RT, } 2 \mathrm{~h}\end{array}$ & ++++ & +++ \\
\hline 8A (see fig. 6) & $\begin{array}{l}1 \% \mathrm{FA}+1 \% \\
\mathrm{AA}\end{array}$ & $20^{\prime}$ & no denaturation & ++++ & +++ \\
\hline $9 A$ & $\begin{array}{l}4 \% \mathrm{FA}+4 \% \\
\mathrm{AA}\end{array}$ & $20^{\prime}$ & no denaturation & +++ & +++ \\
\hline $10 \mathrm{~A}$ & $\begin{array}{l}2 \% \mathrm{GA}+2 \% \\
\mathrm{AA}\end{array}$ & $40^{\prime}$ & no denaturation & + & + \\
\hline $11 \mathrm{~A}$ & $2 \% \mathrm{GA}$ & $\begin{array}{l}\text { 1h } \mathrm{RT} \rightarrow>\text { overnight } \\
\text { at } 4^{\circ} \mathrm{C}\end{array}$ & no denaturation & + & ++ \\
\hline $\begin{array}{l}\text { 12A (see } \\
\text { suppl. fig. 4) }\end{array}$ & $\begin{array}{l}\% \text { FA+ } \\
0.1 \% \text { GA }\end{array}$ & $\begin{array}{l}20^{\prime} \text { (post fix RT, } 10^{\prime} \text {, } \\
0.25 \% \text { GA) }\end{array}$ & no denaturation & ++ & +++ \\
\hline $13 \mathrm{~A}$ & $\begin{array}{l}\text { 3\% Glyoxal+ } \\
3 \% \text { AA }\end{array}$ & $12^{\prime}$ & no denaturation & n. d. & n. d. \\
\hline $14 \mathrm{~A}$ & $\begin{array}{l}\text { 3\% Glyoxal+ } \\
3 \% \text { AA }\end{array}$ & $20^{\prime}$ & no denaturation & n. d. & n. d. \\
\hline $15 A$ & $\begin{array}{l}\text { 3\% Glyoxal+ } \\
3 \% \text { AA }\end{array}$ & $40^{\prime}$ & no denaturation & n. d. & n. d. \\
\hline
\end{tabular}

Monomer solution 2 (MS2)

\begin{tabular}{|c|c|c|c|c|c|}
\hline $\begin{array}{l}\text { Protocol } \\
\text { number }\end{array}$ & Fixative & Time of fixation & Digestion & $\begin{array}{l}\text { Expan } \\
\text { sion }\end{array}$ & $\begin{array}{c}\text { Chromatin } \\
\text { structure }\end{array}$ \\
\hline 1B & $\begin{array}{l}1 \% \mathrm{FA}+1 \% \\
\mathrm{AA}\end{array}$ & $20^{\prime}$ & Proteinase K, RT, 30` & ++++ & ++ \\
\hline $\begin{array}{l}\text { 2B (see suppl. } \\
\text { fig. } 5 \text { ) }\end{array}$ & $\begin{array}{l}1 \% \mathrm{FA}+1 \% \\
\mathrm{AA}\end{array}$ & $20^{\prime}$ & Proteinase $\mathrm{K}, 37^{\circ} \mathrm{C}, 30^{\prime}$ & ++++ & ++ \\
\hline $3 B$ & $\begin{array}{l}1 \% \mathrm{FA}+1 \% \\
\mathrm{AA}\end{array}$ & $20^{\prime}$ & Proteinase $\mathrm{K}, \mathrm{RT}, \mathrm{O} / \mathrm{N}$ & ++++ & ++ \\
\hline $4 B$ & $\begin{array}{l}1 \% \mathrm{FA}+1 \% \\
\mathrm{AA}\end{array}$ & $20^{\prime}$ & $\begin{array}{l}\text { Proteinase } \mathrm{K} \text { buffer, RT, } \\
\text { only } 2 \mathrm{~h}\end{array}$ & ++++ & +++ \\
\hline $\begin{array}{l}5 B \text { (see figs. } \\
4,5)\end{array}$ & $\begin{array}{l}1 \% \mathrm{FA}+1 \% \\
\mathrm{AA}\end{array}$ & $20^{\prime}$ & no digestion & ++++ & ++++ \\
\hline $6 B$ & $\begin{array}{l}4 \% F A+4 \% \\
A A\end{array}$ & $40^{\prime}$ & no digestion & + & ++ \\
\hline $7 B$ & $\begin{array}{l}1 \% \mathrm{FA}+ \\
0.1 \% \mathrm{GA}\end{array}$ & $\begin{array}{l}20^{\prime} \text { (post fix, RT, } 10^{\prime} \\
0.25 \% \text { GA) }\end{array}$ & no digestion & + & +++ \\
\hline
\end{tabular}

The colour code denotes protocols with the same fixation solution. The quality of expansion and chromatin structure is indicated by $+(++++$ the best, + the poorest) 
Fig. 1 Simplified schemata of ExM protocols. The standard ExM protocols for monomer solution 1 and 2 (MS1 and MS2) are shown in (a). The protocols for FISH and indirect immunolabelling were optimised by excluding the denaturation/ digestion steps. Both, MS1 and MS2, can be used (b) a Root fixation, nuclei isolation and nuclei attachment

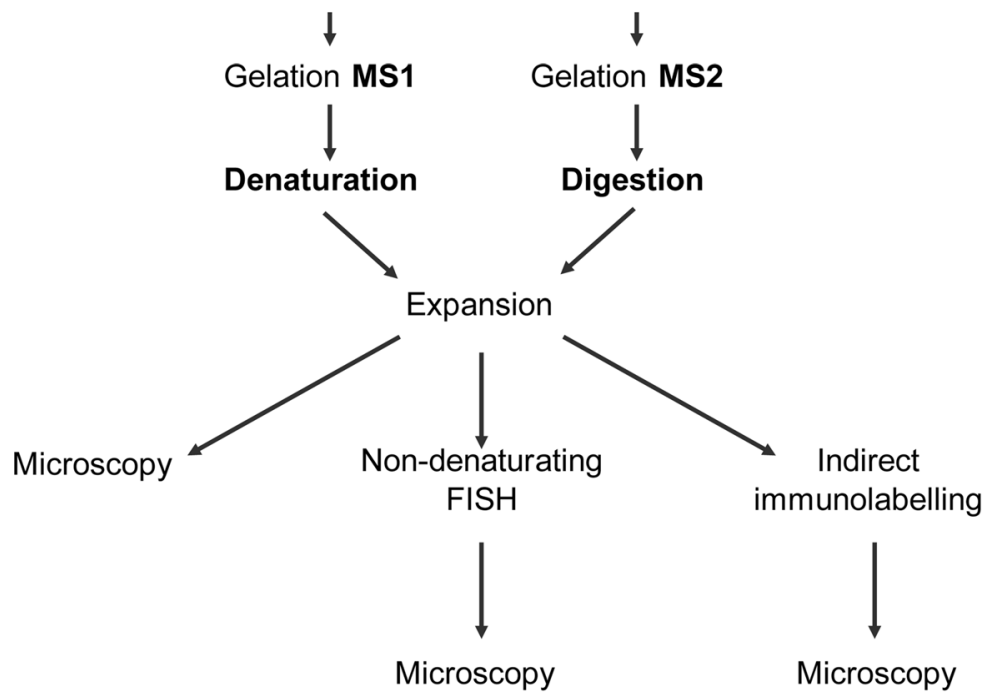

b
Cell Instruments, catalogue no. CM-S22-1). The simplified schema of these protocol steps is shown in Fig. 1 b.

Wide-field, deconvolution and super-resolution microscopy

The chromatin structure was analysed by wide-field (WF), deconvolution (DCV) of WF and super-resolution, using an Elyra PS.1 microscope system and the software ZEN Black (Carl Zeiss GmbH). Images were captured separately for DAPI and Alexa488 using the $405 \mathrm{~nm}$ and $488 \mathrm{~nm}$ laser lines for excitation and appropriate emission filters. To analyse the chromatin ultrastructure at a resolution of $\sim 100 \mathrm{~nm}$ (super-resolution achieved with a $405 \mathrm{~nm}$ laser), structured illumination microscopy (SIM) was performed with a $63 \times / 1.4$ Oil Plan-Apochromat objective (Weisshart et al. 2016).

For SIM imaging of unexpanded and expanded nuclei, a linear grid matching to the respective wavelength was used, and the raw data were processed using the SIM processing function of ZEN Black. First, we started with the automatic mode and then optimized systematically to the highest strength of the Noise Filter where structured noise just disappeared. The resolution in the images was measured with the profile tool of ZEN Black taking a peak-to-peak distance between two structures. In theory, the resolution in the expanded samples could be as high as SIM resolution divided by the 
expansion factor, assuming that the sample expansion was isotropic. The procedure to process the SIM raw data and to estimate the achieved resolution of unexpanded and expanded nuclei after WF and SIM imaging is described in detail in Kubalová et al. (2020).

The WF and deconvoluted WF images were calculated in parallel to SIM processing by ZEN Black.

\section{Results}

A 4.2-times expansion of isolated plant nuclei can be achieved without denaturation and digestion

To establish a protocol to expand isolated interphase nuclei while preserving the chromatin ultrastructure, several fixation conditions and two different monomer solution (MS) compositions were tested for expansion microscopy (ExM) (Table 1). To analyse the effect of expansion on the chromatin ultrastructure, we applied wide-field (WF), deconvolution (DCV) of WF images and structured illumination microscopy (SIM). Unexpanded nuclei were imaged by all three techniques as the untreated control to evaluate whether the imaging of expanded nuclei by WF could deliver structural information comparable to DCV or SIM applied to unexpanded nuclei (Fig. 2). Additionally, SIM on expanded nuclei was applied to assess whether the chromatin ultrastructure was altered due to the swelling of nuclei (Figs. 3, 4, 5 and 6).

First, we started with monomer solution 1 (MS1) with a higher concentration of acrylamide (Table 1) and performed 12-min fixation of barley root tips in a mixture of $2 \%$ formaldehyde (FA) $+2 \%$ acrylamide (AA) in $1 \times$ PBS prior the extraction of nuclei. To homogenize the specimens, we applied heat denaturation for $5 \mathrm{~min}$ at $96^{\circ} \mathrm{C}$ in denaturation buffer containing SDS (protocol variant 2A in Table 1). Although we observed expanded nuclei, the chromatin structure was poor. Therefore, we reduce time and temperature of denaturation in denaturation buffer containing SDS for $3 \mathrm{~min}$ at $80{ }^{\circ} \mathrm{C}$ (protocol variant $2 \mathrm{~B}$ in Table 1 ).

After expansion, we observed a $\sim 4.2$-times expansion of the gel and nuclei (Fig. 3; Suppl. Fig. 1). As the size of untreated nuclei varied between 9 and $25 \mu \mathrm{m}$ in diameter due to the mixed tissue-type origin (tissue layers of the root tip) and status of DNA replication (G1, S and G2), also the size of expanded nuclei varied between $\sim 40$ and $\sim 110 \mu \mathrm{m}$ (Suppl. Fig. 2).
By using a $\times 20 / 0.8$ objective, we observed that all nuclei were expanded and showed similar structures (Suppl. Fig. 2). A few of them were selected for SIM analysis using the $\times 63 / 1.4$ objective. Structural features, such as the nucleus shape and nucleoli, were always visible after expansion. Also, the chromatin arrangement into distinct domains representing chromatin associations became visible by WF (Suppl. Figs. 1a, b). However, the application of DCV and SIM revealed that the chromatin ultrastructure was impaired because chromatin fibres organized as a network and clearly visible in unexpanded nuclei by SIM (Fig. 2e, f), were not detectable (Suppl. Fig. 1e, f).

To improve the expansion protocol, we denatured the specimen for 3 min with $0.2 \mathrm{M} \mathrm{NaOH}$ in $70 \%$ ethanol, instead of heat denaturation. The alkaline/ethanol denaturation is commonly used to denature DNA during the FISH procedure and is superior for chromatin structure preservation (Raap et al. 1986; Andras et al. 1999). Again, we achieved a 4.2-times expansion of gel and nuclei. All microscopical imaging methods showed a better preserved chromatin ultrastructure of expanded nuclei. Even the application of WF microscopy showed structures like chromatin domains with a resolution of $\sim 50-60 \mathrm{~nm}$ that are invisible in unexpanded nuclei (Suppl. Figs. 3a-c; protocol variant $3 \mathrm{~A}$ in Table 1). To achieve an optimal SIM image quality, the moderate noise filter setting of -3.8 was applied in the SIM calculation tool of the ZEN Black software. A filter setting of 1.0 representing a high strength for the calculation was only suitable for the calculation of unexpanded nuclei (Weisshart et al. 2016). Thus, expansion leads to a decrease in the signal-to-noise ratio (SNR) of the images, and hence to the reduction of the specific sample fluorescence. Nevertheless, in expanded nuclei, SIM imaging resulted in a resolution of $\sim 25-35 \mathrm{~nm}$.

Next, to simplify the protocol and further improve the chromatin ultrastructure preservation, we omitted the denaturation step (protocol variant 4A in Table 1). However, the chromatin structure was not improved compared with the $0.2 \mathrm{M} \mathrm{NaOH}$ in $70 \%$ ethanol denaturation.

The expansion of nuclei is a physical process that might alter the native structure of chromatin when nuclei become strongly fixed. Therefore, we tested whether a 

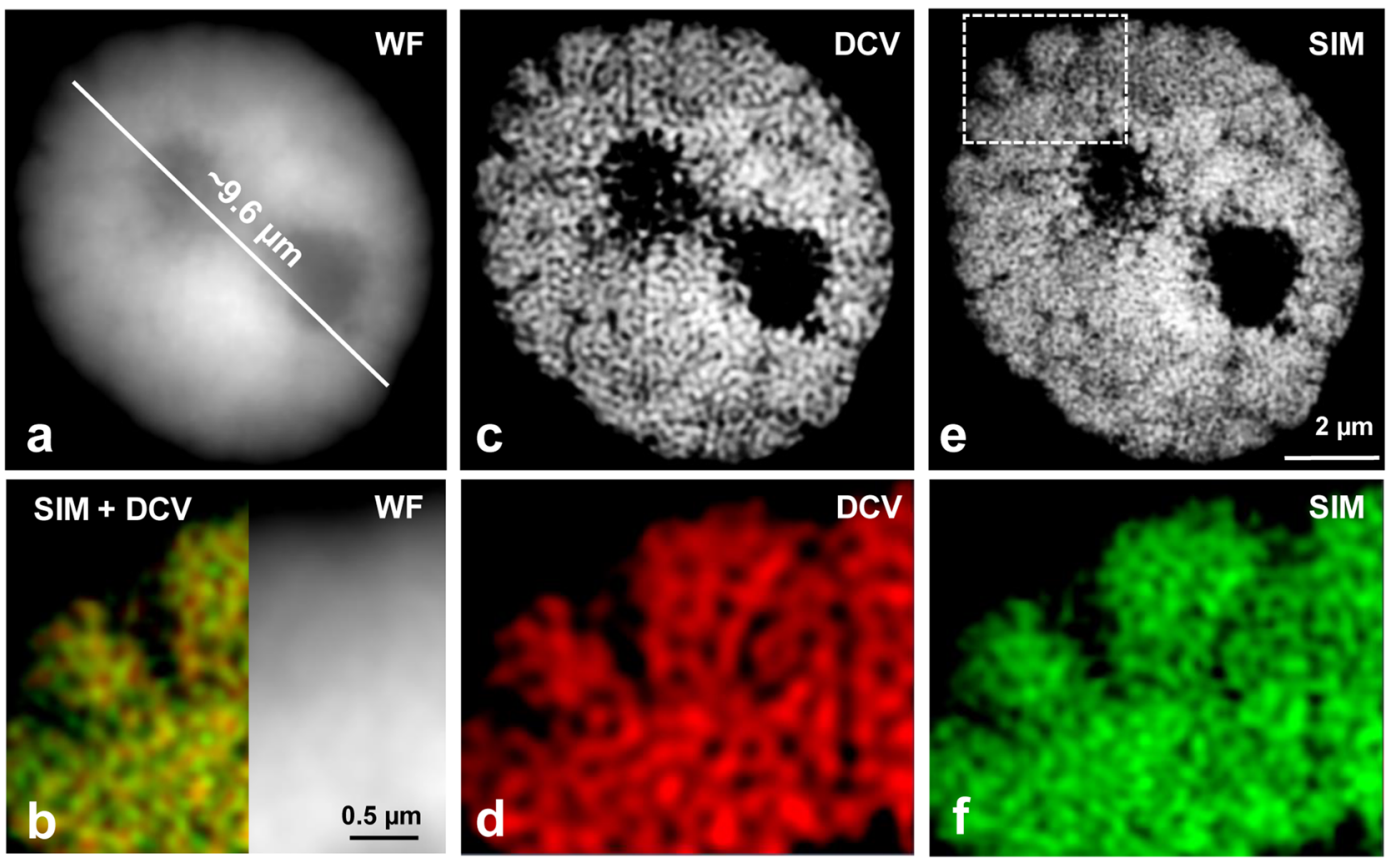

Fig. 2 The chromatin ultrastructure is well maintained in an unexpanded nucleus, as especially visible after structured illumination microscopy (SIM) compared with deconvolution (DCV) and wide-field microscopy (WF). Despite the different achieved resolution (a, c, e), the merge (b, left) of DCV (c) and SIM (e)

gentle fixation of root tips in $1 \% \mathrm{FA}+1 \%$ AA could improve the preservation of the chromatin structure. First, the short 12-min fixation in $1 \% \mathrm{FA}+1 \% \mathrm{AA}$, no denaturation, had a small impact on chromatin structure improvement. Therefore, we prolonged the fixation time for $20 \mathrm{~min}$ (protocol variant $8 \mathrm{~A}$ in Table 1). This condition delivered a similar chromatin structure as the $0.2 \mathrm{M} \mathrm{NaOH}$ in $70 \%$ ethanol denaturation.

Additionally, we tried a combination of $1 \% \mathrm{FA}$ and $1 \%$ glutaraldehyde as fixation solution and applied $0.25 \%$ glutaraldehyde for post-fixation (protocol variant $12 \mathrm{~A}$ in Table 1). Again, we omitted the denaturation step, since it may impair the chromatin structure. However, the tested fixation conditions did not improve the preservation of the chromatin structure. Moreover, we observed nuclei less expanded ( $47 \mu \mathrm{m}$; (Suppl. Fig. 4) than nuclei fixed in a FA/AA mixture $(\sim 61 \mu \mathrm{m}$; Fig. 6h). Thus, the applied fixation solutions influence the ability of the nuclei to expand, and the fixation time can affect the chromatin structure. Hence, the fixation in a mixture of $1 \% \mathrm{FA}$ and $1 \% \mathrm{AA}$ for a prolonged time of $20 \mathrm{~min}$ is most suitable for ExM.

After applying the third fixation solution, glyoxal, we could not detect nuclei within the gel. indicates in the enlarged region (dashed rectangle) $(\mathbf{b}, \mathbf{d}, \mathbf{f})$ that the same chromatin structures were identified. Instead, no clear structures are visible in the zoomed region by WF (b, right). Global chromatin was labelled by DAPI

Besides the original MS1, we used MS2 with less acrylamide concentration (Table 1). We performed an enzymatic digestion of nuclei using proteinase $\mathrm{K}$ (protocol variants 1B-3B in Table 1). Using both MS1 and MS2, expanded nuclei were observed, but the chromatin structure was better preserved by MS2 (Suppl. Fig. 5). In addition to chromatin, also nucleoli were strongly labelled by DAPI suggesting that the proteinase $\mathrm{K}$ treatment may have impaired the chromatin stability. Hence, we omitted the digestion with proteinase $\mathrm{K}$ from the protocol (protocol variant 5B in Table 1). Thus, we obtained expanded nuclei with chromatin structures already visible by WF, but with nucleoli free of DAPI labelling (Fig. 4a, d, g). SIM, DCV and WF showed comparable images. Further magnification revealed network-like structures in all three imaging methods (Fig. 4b-i). The protocols delivering less expanded nuclei or very little preserved chromatin were omitted from the further optimization process. The protocols $1 \% \mathrm{FA} / \mathrm{AA}$ in MS1 or MS2, no denaturation/ digestion (variants $8 \mathrm{~A}$ or $5 \mathrm{~B}$ in Table 1) showing the best-expanded nuclei were applied in three independent experiments. 
Fig. 3 The expansion factor of gel (a) and nuclei (b) correspond to $\sim 4.2$. Nuclei were stained by DAPI and imaged by structured illumination microscopy (SIM), deconvolution (DCV) and widefield microscopy (WF). Imaged by WF, the expanded nucleus shows more structures than the original one, but does not reach the resolution of SIM
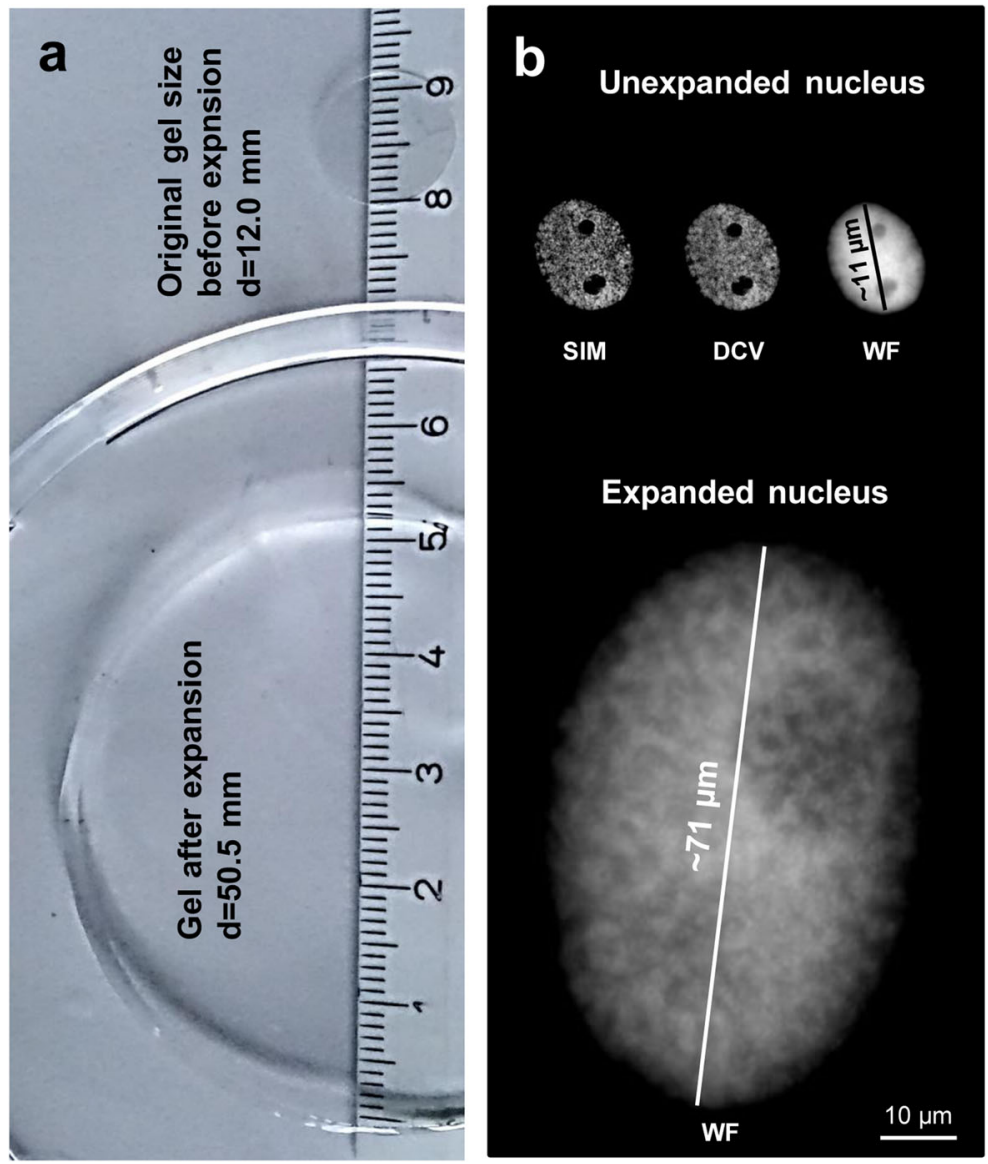

Except for their ultrastructure, the global chromatin arrangement of expanded nuclei is preserved after ND-FISH and immunolabelling

Expansion microscopy in combination with nondenaturating fluorescence in situ hybridization (NDFISH) for the visualization of high-copy repeats in expanded nuclei was tested next. A DNA denaturation step, an essential part of the standard FISH procedure, was omitted since it was shown that the detection of barley centromere and telomere repeats does not require this step (Cuadrado et al. 2009). Using a barley centromere-specific probe, we detected by WF microscopy in expanded nuclei hybridization signals corresponding to centromeres arranged in Rabl orientation, which originates from the former arrangement of the centromeres during telophase (Rabl 1885), an interphase organization also common for barley (Schubert et al. 2016) (Fig. 5). However, SIM revealed that the arrangement of chromatin in fibres and domains was damaged
(Fig. $5 \mathrm{j}-1$ ). Thus, the position of repeats after nuclei expansion was maintained, but the chromatin ultrastructure organized in a network-like manner was lost. The overall quality of the chromatin structure was low compared with the protocol without ND-FISH, presumably due to the additional treatment steps required for NDFISH. The overnight incubation in 50\% deionized formamide could have a negative impact on the preservation of chromatin.

To investigate whether proteins can be visualized in expanded barley nuclei, we employed indirect immunolabelling using antibodies against the centromere-specific histone variant $\mathrm{CENH} 3$ of barley (Houben et al. 2007). In contrast to FISH, instead of monomer solution MS2, we used solution MS1. Since indirect immunolabelling requires several incubation and re-expansion steps and MS2 delivers fragile gels due to the low acrylamide amount, it is challenging to conduct the protocol without damaging the gel. The immunofluorescence signals of CENH3 could be 

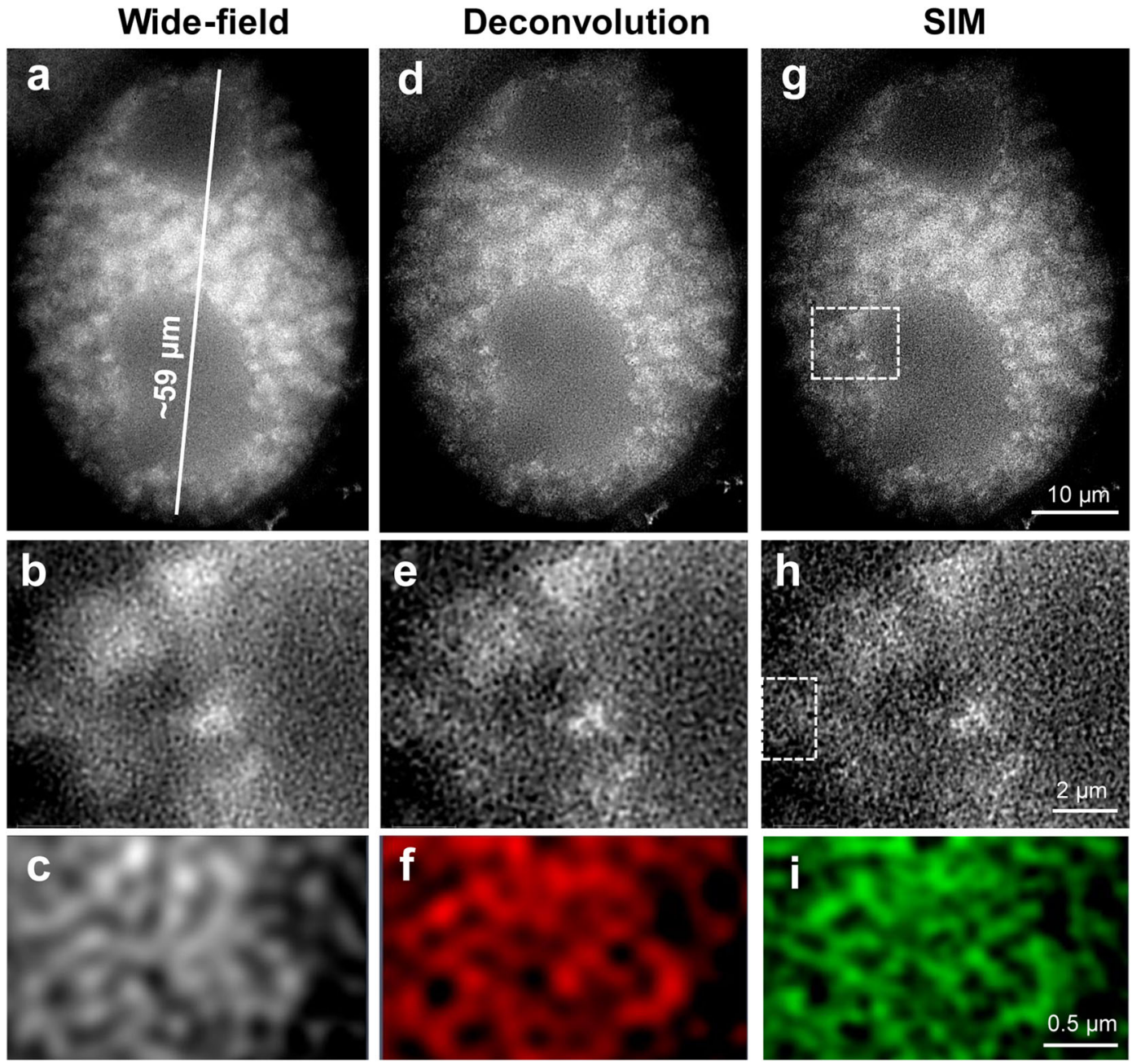

Fig. 4 Well-preserved chromatin structure achieved by application of ExM protocol variant 5B (see Table 1). The subsequent magnifications of the selected regions (dashed rectangles) demonstrate the preserved chromatin ultrastructure visible even after

detected independently of the used monomer solution. Prior to expansion, no denaturation/digestion was carried out.

Similar to the centromere-specific repeats identified by FISH, the CENH3 signals displayed the expected Rabl-like positions in expanded and unexpanded nuclei (Fig. 6). Imaged by WF, the immunosignals were homogeneous. But SIM revealed that the CENH3 signals were scattered in expanded nuclei (Fig. 6j). In contrast, the CENH3 and overall chromatin structures of unexpanded nuclei were well preserved (Fig. 6d-f).

In summary, ExM allows enlarging isolated plant nuclei physically with an expansion factor of $\sim 4.2$ times. Barley nuclei with a size between $\sim 40$ and $110 \mu \mathrm{m}$ were wide-field microscopy $(\mathbf{a}, \mathbf{b}, \mathbf{c})$ of this completely expanded nucleus labelled by DAPI. By comparing WF with deconvolution (d, e, f) and $\operatorname{SIM~(g,~h,~i),~similar~chromatin~structures~become~visible~}$ at the highest magnification (region $90^{\circ}$ rotated)

obtained. Distinct chromatin domains can be detected in expanded nuclei without the need for optical superresolution microscopy (Fig. 4). Barley centromeres can be visualized by combining ExM with ND-FISH or indirect immunolabelling. However, super-resolution microscopy revealed that ExM results in the impairment of the network-like organization of the chromatin substructure.

\section{Discussion}

We applied ExM to isolated plant nuclei. With the aim to preserve the chromatin structure, we adapted 

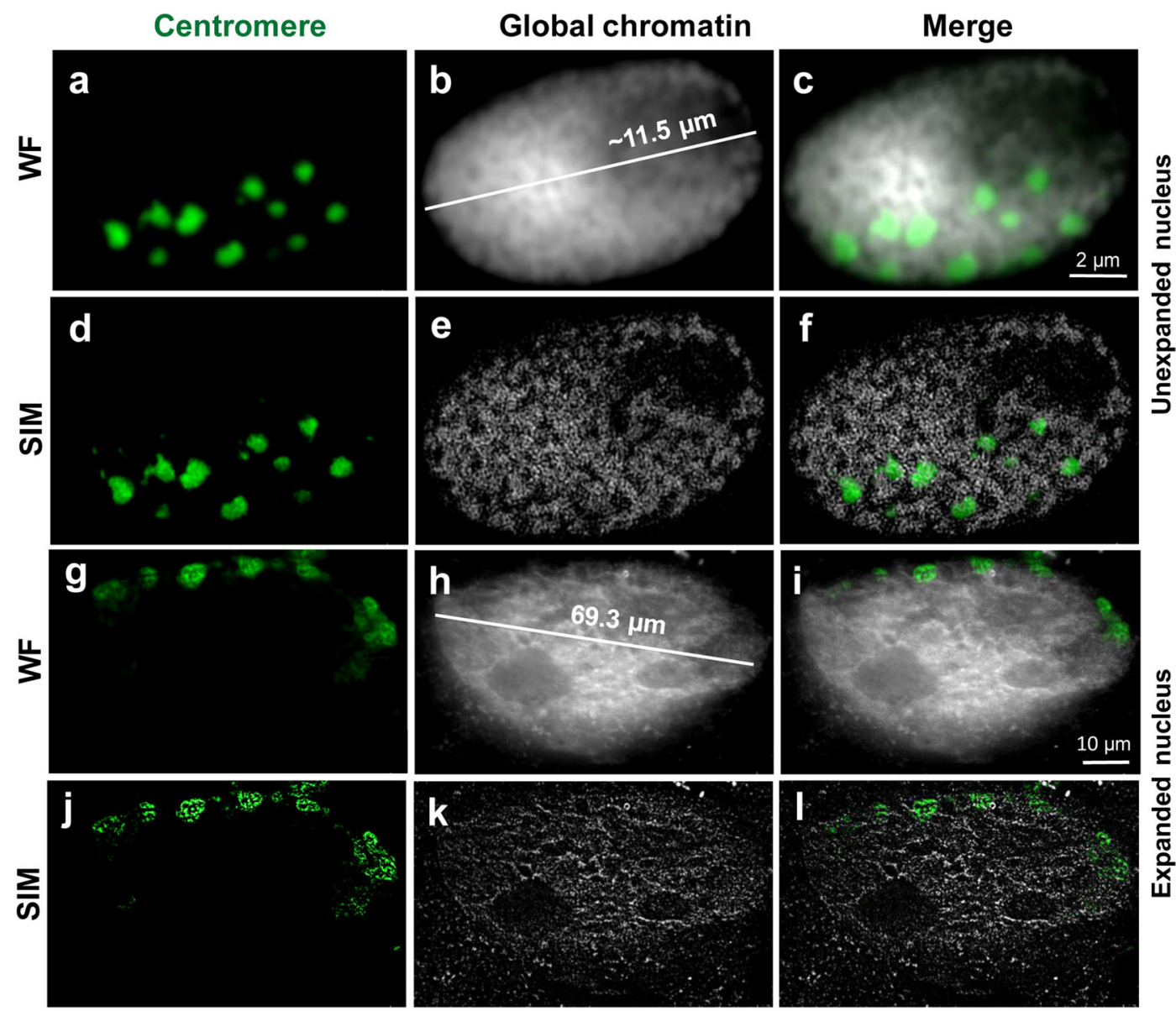

Fig. 5 FISH detection of centromeric repeats $(\mathrm{GA})_{15}$ at unexpanded (a-f) and expanded ( $\mathbf{g}-\mathbf{l})$ nuclei using ExM protocol variant 5B (see. Table 1). Although the main centromeric structures are maintained after complete expansion and thus indicating

the U-ExM protocol of Gambarotto et al. (2019) which was initially established for centrioles of Chlamydomonas. To ensure the best possible chromatin structure maintenance we tested different fixatives such as formaldehyde, glutaraldehyde, and glyoxal. It is known that fixation has a crucial effect on chromatin structure preservation (Kozubek et al. 2000; Howat and Wilson 2014). Guillot et al. (2004) demonstrated that fixation and cell permeabilization affects the distribution of RNA polymerase II molecules in human cells under conditions that do not sustain the cellular ultrastructure. While formaldehyde is routinely used for the fixation of specimens before immunolabelling and light microscopy (Puchtler and Meloan 1985), glutaraldehyde is commonly used for electron microscopy-based observations (Hayat 1986; Park et al. 2016). Glyoxal was successfully applied for different animal tissues to improve
Rabl orientation, WF (g, h, i) and SIM (j, $\mathbf{k}, \mathbf{l})$ imaging shows that the chromatin ultrastructures are impaired. Global chromatin was labelled by DAPI

structural features and to reduce formaldehyde fixation artefacts (Richter et al. 2018). Fixation procedures required before applying immunolabelling and FISH may induce structural artefacts within the specimens. However, Markaki et al. (2012) demonstrated that by appropriately adapting 3D-FISH, the key characteristics of cell nuclei are preserved and that SIM discovers new insights into the functional nuclear organization.

Although all fixatives kept nuclear morphology and the nucleoli of barley nuclei, only formaldehyde allowed the expansion of $\sim 4.2$ times. The mild fixation of roots using $1 \% \mathrm{FA}$ and $1 \%$ AA with a $20-\mathrm{min}$ incubation time provided better results than the stronger fixation of roots in $4 \% \mathrm{FA}+4 \% \mathrm{AA}$ for 20 or $40 \mathrm{~min}$. The sample preparation steps, denaturation and digestion, which are required to homogenize the mechanical properties of different non-plant tissues (Chen et al. 

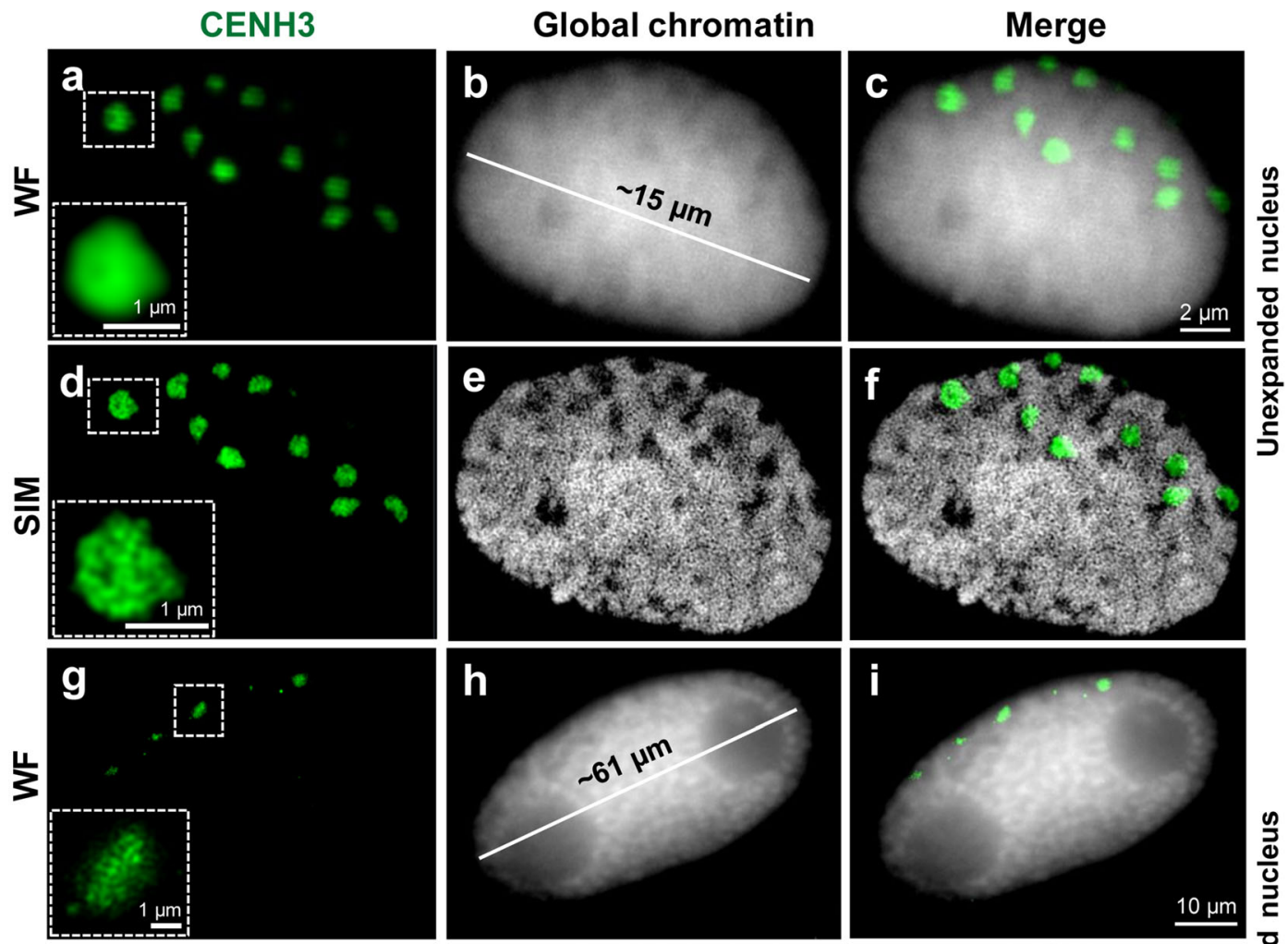

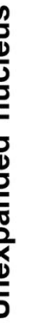
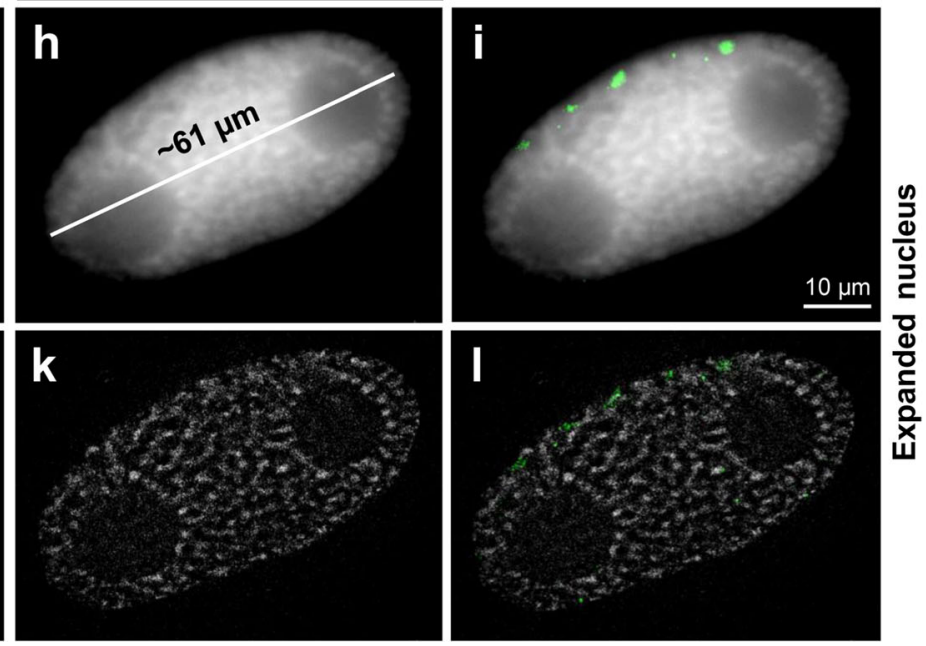

Fig. 6 Detection of the centromere-specific histone variant CENH3 after immunolabelling and ExM according to protocol variant $8 \mathrm{~A}$ (see Table 1). Although the main centromeric structures are preserved after complete expansion, SIM and WF imaging indicates that the chromatin ultrastructures are impaired. The

2015; Cho et al. 2018; Wassie et al. 2019) were omitted because both steps impaired strongly the chromatin structure. This observation is in agreement with previous studies showing that chromatin becomes damaged when isolated nuclei were exposed to a detergent (Szabó et al. 1990). A similar negative effect has been described for proteinase $\mathrm{K}$ that cleaves chromatin into $50-\mathrm{kb}$ fragments (Szabó et al. 1990; Gal et al. 2000). We speculate that this might be the reason that DAPI-specific signals were found within the nucleolus after proteinase $\mathrm{K}$ treatment. Further, DNA damage leads to the accumulation of RNAs and proteins inside the nucleolus (Lindström and Latonen 2013; Jin et al. 2014). enlarged region (dashed rectangle) shows clearly the preserved and distorted CENH3-positive chromatin in an unexpanded (a-f) and expanded (g-l) nucleus, respectively. Global chromatin was labelled by DAPI

Moreover, Kao and Nodine (2019) showed that a mild proteinase $\mathrm{K}$ treatment impairs the fluorescence signal intensities after immunolabelling in expanded Arabidopsis ovules and seeds. Thus, we conclude that protocol variants 5B and 8A shown in Table 1 are the most suitable for ExM of isolated barley nuclei. Noteworthy, these variants do not include a homogenisation process. If this step is required, denaturation with $0.2 \mathrm{M}$ $\mathrm{NaOH}$ in $70 \%$ ethanol is recommended.

ExM allowed us to visualize the main structural features of nuclei-like nuclear shape and the nucleoli and to observe chromatin structures invisible in unexpanded nuclei when detected with classical WF 
microscopy. To further increase the resolution of expanded specimens and to check the substructure maintenance of chromatin, we applied super-resolution microscopy after ExM. We identified network-like organized chromatin, similar to that observed in unexpanded nuclei of mammals (Markaki et al. 2012) and plants (Ma et al. 2017; Schubert 2017).

However, the combination of ExM with SIM did not result in more structural information, although the achieved SIM resolution $(\sim 25-35 \mathrm{~nm})$ in expanded nuclei was higher than in unexpanded ones $(\sim 50$ $60 \mathrm{~nm}$ ). Instead, the visualization of chromatin in unexpanded nuclei by SIM delivers better results (compare Figs. 2 and 4). What could be the reason? Pernal et al. (2020) showed that expansion is anisotropic not only between different tissues but also between different subcellular compartments and even within subcellular compartments themselves. This observation may be a reason that the chromatin ultrastructure within the nuclei becomes damaged and is difficult to preserve even after appropriate fixation. The employed $63 \times / 1.4$ oil objective (working distance $0.19 \mathrm{~mm}$ ) required for SIM caused another technical challenge due to the impossibility to analyse nuclei which were distantly embedded from the coverslip. To circumvent this problem, Cahoon et al. (2017) prepared cryosections and successfully observed the expanded synaptonemal complex of the fruit fly. But this approach is laborious and not suitable for high-throughput experiments.

We combined ExM with ND-FISH to detect centromeric repeats in barley. The position of the detected fluorescence signals corresponded to centromeric signals observed in original, unexpanded nuclei. Thus, a nucleus that underwent expansion maintains its general morphology and chromatin organization. On the other hand, SIM revealed that the ultrastructure of centromeric chromatin was only partially preserved after ExM and ND-FISH. The combination of ExM and FISH was successfully used to visualize mRNA in expanded mammalian cell cultures and brain tissue (Chen et al. 2016; Wang et al. 2018a), but their protocols differ from our protocols by using different fixation solution and omitting the denaturation/digestion steps. Moreover, compared with the relatively short mRNA molecules, chromatin is more complex by creating network-like structures in animals and plants (Markaki et al. 2012; Schubert 2014; Beseda et al. 2020) and thus can collapse more easily during the process of physical magnification. Therefore, we speculate that current ExM protocols can reveal and detect the correct RNA positions using FISH, but sustaining the chromatin ultrastructure is more challenging. Similar to the FISH experiments, we localized the centromere-specific protein CENH3 in the correct Rabl orientation, but again, the chromatin ultrastructure was impaired. This observation is reasonable because $\mathrm{CENH} 3$, as a component of nucleosomes, is associated with DNA-forming centromeric heterochromatin.

On the other hand, several reports (Chozinski et al. 2016; Cahoon et al. 2017; Freifiled et al. 217; Jiang et al. 2018; Wang et al. 2018b; Gambarotto et al. 2019; Kao and Nodine 2019; Xu et al. 2019; Zwettler et al. 2020a, b) demonstrated the improved visualisation of target proteins after applying ExM. Thus, ExM can reveal the unaltered localization of target molecules, but preserving the chromatin ultrastructure of isolated nuclei is more challenging, and therefore, further improved ExM protocols have to be developed. Otherwise, expanded chromatin structures imaged by wide-field microscopy will not deliver more information as achieved by superresolution microscopy on unexpanded structures.

Funding Open Access funding provided by Projekt DEAL. This work has been supported by the Deutsche Forschungsgemeinschaft (Schu 762/11-1) and by the Czech Science Foundation (17-20613Y).

Abbreviations 3D, 3-dimensional; $A A$, Acrylamide; APS, Ammonium persulfate; DAPI, 4',6-diamidino-2phenylindole; $D C V$, Deconvolution; FA, Formaldehyde; $N D$-FISH, Non-denaturating Fluorescence in situ hybridization; GA, Glutaraldehyde; $h$, Hour; min, Minutes; $M S$, Monomer solution; $n$. d., not determined; $P B S$, Phosphate buffer saline; $P L L$, Poly-L-lysine; $R T$, Room temperature; $S I M$, Structured illumination microscopy; TEMED,

Tetramethylethylenediamine; U-ExM, Ultrastructure expansion microscopy; $W F$, Wide-field

Open Access This article is licensed under a Creative Commons Attribution 4.0 International License, which permits use, sharing, adaptation, distribution and reproduction in any medium or format, as long as you give appropriate credit to the original author(s) and the source, provide a link to the Creative Commons licence, and indicate if changes were made. The images or other third party material in this article are included in the article's Creative Commons licence, unless indicated otherwise in a credit line to the material. If material is not included in the article's Creative Commons licence and your intended use is not permitted by statutory regulation or exceeds the permitted use, you will need to obtain permission directly from the copyright holder. To view a copy of this licence, visit http://creativecommons.org/licenses/by/4.0/. 


\section{References}

Alon S, Huynh GH, Boyden ES (2019) Expansion microscopy: enabling single cell analysis in intact biological systems. FEBS J 286:1482-1494

Andras SC, Hartman TPV, Marshall JA, Marchant R, Power JB, Cocking EC, Davey MR (1999) A drop-spreading technique to produce cytoplasm-free mitotic preparations from plants with small chromosomes. Chromosom Res 7:641-647

Asano SM, Gao R, Wassie AT, Tillberg PW, Chen F, Boyden ES (2018) Expansion microscopy: protocols for imaging proteins and RNA in cells and tissues. Current Protoc Cell biol 80:e56

Beseda T, Cápal P, Kubalová I, Schubert V, Doležel J, Šimková H (2020) Mitotic chromosome organization: general rules meet species-specific variability. Comput Struct Biotech J 18: 1311-1319

Cahoon CK, Yu Z, Wang Y, Guo F, Unruh JR, Slaughter BD, Hawley RS (2017) Superresolution expansion microscopy reveals the three-dimensional organization of the Drosophila synaptonemal complex. Proc Natl Acad Sci USA 114:e6857-e6866

Chang JB, Chen F, Yoon YG, Jung EE, Babcock H, Kang JS, Asano S, Suk HJ, Pak N, Tillberg PW, Wassie AT, Cai D, Boyden ES (2017) Iterative expansion microscopy. Nat Methods 14:593-599

Chen F, Tillberg PW, Boyden ES (2015) Expansion microscopy. Science 347:543-548

Chen F, Wassie AT, Cote AJ, Sinha A, Alon S, Asano S, Daugharthy ER, Chang JB, Marblestone A, Church GM, Raj A, Boyden ES (2016) Nanoscale imaging of RNA with expansion microscopy. Nat Methods 13:679-684

Cho I, Seo JY, Chang J (2018) Expansion microscopy. J Microsc 271:123-128

Chozinski TJ, Halpern AR, Okawa H, Kim HJ, Tremel GJ, Wong RO, Vaughan JC (2016) Expansion microscopy with conventional antibodies and fluorescent proteins. Nat Methods $13: 485-488$

Cuadrado Á, Golczyk H, Jouve N (2009) A novel, simple and rapid nondenaturing FISH (ND-FISH) technique for the detection of plant telomeres. Potential used and possible target structures detected. Chromosome Res 17:755

Doležel J, Greilhuber J, Suda J (2007) Estimation of nuclear DNA content in plants using flow cytometry. Nat Protoc 2:22332244

Düring DN, Rocha MD, Dittrich F, Gahr M, Hahnloser RHR (2019) Expansion light sheet microscopy resolves subcellular structures in large portions of the songbird brain. Front Neuroanat 13:2

Endo TR, Kubalakova M, Vrana J, Dolezel J (2014) Hyperexpansion of wheat chromosomes sorted by flow cytometry. Genes Genet Syst 89:181-185

Fornasiero EF, Opazo F (2015) Super-resolution imaging for cell biologists: concepts, applications, current challenges and developments. BioEssays 37:436-451

Freifeld L, Odstrcil I, Forster D, Ramirez A, Gagnon JA, Randlett O, Costa EK, Asano S, Celiker OT, Gao R, Martin-Alarcon DA, Reginato P, Dick C, Chen L, Schoppik D, Engert F, Baier H, Boyden ES (2017) Expansion microscopy of zebrafish for neuroscience and developmental biology studies. Proc Natl Acad Sci USA 114:e10799-e10808

Gal I, Varga T, Szilagyi I, Balazs M, Schlammadinger J, Szabo G Jr (2000) Protease-elicited TUNEL positivity of nonapoptotic fixed cells. J Histochem Cytochem 48:963-970

Gambarotto D, Zwettler FU, Le Guennec M, SchmidtCernohorska M, Fortun D, Borgers S, Heine J, Schloetel JG, Reuss M, Unser M, Boyden ES, Sauer M, Hamel V, Guichard P (2019) Imaging cellular ultrastructures using expansion microscopy (U-ExM). Nat Methods 16:71-74

Götz R, Panzer S, Trinks N, Eilts J, Wagener J, Turra D, Di Pietro A, Sauer M, Terpitz U (2020) Expansion microscopy for cell biology analysis in fungi. Front Microbiol 11:574

Guillot PV, Xie SQ, Hollinshead M, Pombo A (2004) Fixationinduced redistribution of hyperphosphorylated RNA polymerase II in the nucleus of human cells. ExpCell Research 295:460-468

Halpern AR, Alas GCM, Chozinski TJ, Paredez AR, Vaughan JC (2017) Hybrid structured illumination expansion microscopy reveals microbial cytoskeleton organization. ACS Nano 11: 12677-12686

Hayat MA (1986) Glutaraldehyde: role in electron microscopy. Micron Microsc Acta 17:115-135

Houben A, Schroeder-Reiter E, Nagaki K, Nasuda S, Wanner G, Murata M, Endo TR (2007) CENH3 interacts with the centromeric retrotransposon cereba and GC-rich satellites and locates to centromeric substructures in barley. Chromosoma 116:275-283

Howat WJ, Wilson BA (2014) Tissue fixation and the effect of molecular fixatives on downstream staining procedures. Methods 70:12-19

Jiang N, Kim HJ, Chozinski TJ, Azpurua JE, Eaton BA, Vaughan JC, Parrish JZ (2018) Superresolution imaging of Drosophila tissues using expansion microscopy. Mol Biol Cell 29:14131421

Jin Y-Q, An G-S, Ni J-H, Li S-Y, Jia H-T (2014) ATM-dependent E2F1 accumulation in the nucleolus is an indicator of ribosomal stress in early response to DNA damage. Cell Cycle 13:1627-1638

Kao P, Nodine MD (2019) Transcriptional activation of Arabidopsis zygotes is required for initial cell divisions. Sci Rep 9:17159

Komis G, Samajova O, Ovecka M, Samaj J (2015) Superresolution microscopy in plant cell imaging. Trends Plant Sci 20:834-843

Kozubek S, Lukasova E, Amrichova J, Kozubek M, Liskova A, Slotova J (2000) Influence of cell fixation on chromatin topography. Anal Biochem 282:29-38

Kubalová I, Schmidt Černohorská M, Huranová M, Klaus Weisshart K, Houben A, Schubert V (2020) A protocol to expand plant nuclei. Methods Cell Biol, https://doi. org/10.1016/bs.mcb.2020.06.007

Lim Y, Shiver AL, Khariton M, Lane KM, Ng KM, Bray SR, Qin J, Huang KC, Wang B (2019) Mechanically resolved imaging of bacteria using expansion microscopy. PLOS Biol 17: e3000268

Lindström MS, Latonen L (2013) The nucleolus as a stress response organelle. In: O'Day DH, Catalano A (eds) Proteins of the nucleolus: regulation, translocation, \& biomedical functions. Springer Netherlands, Dordrecht, pp 251-273 
Ma W, Gabriel TS, Martis MM, Gursinsky T, Schubert V, Vrána J, Doležel J, Grundlach H, Altschmied L, Scholz U, Himmelbach A, Behrens S-E, Banaei-Moghaddam AM, Houben A (2017) Rye B chromosomes encode a functional Argonaute-like protein with in vitro slicer activities similar to its A chromosome paralog. New Phytol 213:916-928

Markaki Y, Smeets D, Fiedler S, Schmid VJ, Schermelleh L, Cremer T, Cremer M (2012) The potential of 3D-FISH and super-resolution structured illumination microscopy for studies of 3D nuclear architecture: 3D structured illumination microscopy of defined chromosomal structures visualized by $3 \mathrm{D}$ (immuno)-FISH opens new perspectives for studies of nuclear architecture. BioEssays 34:412-426

Park C-H, Kim H-W, Rhyu IJ, Uhm C-S (2016) How to get wellpreserved samples for transmission electron microscopy. AM 46:188-192

Pernal SP, Liyanaarachchi A, Gatti DL, Formosa B, Pulvender R, Kuhn ER, Ramos R, Naik AR, George K, Arslanturk S, Taatjes DJ, Jena BP (2020) Nanoscale imaging using differential expansion microscopy. Histochem Cell Biol 153:469480

Puchtler H, Meloan SN (1985) On the chemistry of formaldehyde fixation and its effects on immunohistochemical reactions. Histochemistry 82:201-204

Raap AK, Marijnen JG, Vrolijk J, van der Ploeg M (1986) Denaturation, renaturation, and loss of DNA during in situ hybridization procedures. Cytometry 7:235-242

Rabl C (1885) Über Zelltheilung. Morph Jb 10: 214-330

Richter KN, Revelo NH, Seitz KJ, Helm MS, Sarkar D, Saleeb RS, D'Este E, Eberle J, Wagner E, Vogl C, Lazaro DF, Richter F, Coy-Vergara J, Coceano G, Boyden ES, Duncan RR, Hell SW, Lauterbach MA, Lehnart SE, Moser T, Outeiro TF, Rehling P, Schwappach B, Testa I, Zapiec B, Rizzoli SO (2018) Glyoxal as an alternative fixative to formaldehyde in immunostaining and super-resolution microscopy. EMBO J $37: 139-159$

Schubert V (2014) RNA polymerase II forms transcription networks in rye and Arabidopsis nuclei and its amount increases with endopolyploidy. Cytogenet Genome Res 143:69-77

Schubert V (2017) Super-resolution microscopy - applications in plant cell research. Front Plant Sci 8:531

Schubert V, Ruban A, Houben A (2016) Chromatin ring formation at plant centromeres. Front Plant Sci 7:28

Schubert V, Weisshart K (2015) Abundance and distribution of RNA polymerase II in Arabidopsis interphase nuclei. J Exp Bot 66:1687-1698

Tillberg PW, Chen F (2019) Expansion microscopy: scalable and convenient super-resolution microscopy. Ann Rev Cell Dev Biol 35:683-701

Tillberg PW, Chen F, Piatkevich KD, Zhao Y, Yu C-C, English BP, Gao L, Martorell A, Suk H-J, Yoshida F, DeGennaro EM, Roossien DH, Gong G, Seneviratne U, Tannenbaum
SR, Desimone R, Cai D, Boyden ES (2016) Protein-retention expansion microscopy of cells and tissues labeled using standard fluorescent proteins and antibodies. Nat Biotechnol 34:987-992

Truckenbrodt S, Sommer C, Rizzoli SO, Danzl JG (2019) A practical guide to optimization in X10 expansion microscopy. Nat Protoc 14:832-863

Wang G, Moffitt JR, Zhuang X (2018a) Multiplexed imaging of high-density libraries of RNAs with MERFISH and expansion microscopy. Sci Rep 8:4847

Wang Y, Yu Z, Cahoon CK, Parmely T, Thomas N, Unruh JR, Slaughter BD, Hawley RS (2018b) Combined expansion microscopy with structured illumination microscopy for analyzing protein complexes. Nat Protoc 13:1869-1895

Wassie AT, Zhao Y, Boyden ES (2019) Expansion microscopy: principles and uses in biological research. Nat Methods 16: 33-41

Weisshart K, Fuchs J, Schubert V (2016) Structured illumination microscopy (SIM) and photoactivated localization microscopy (PALM) to analyze the abundance and distribution of RNA polymerase II molecules on flow-sorted Arabidopsis nuclei. Bio-protocol 6:e1725

Xu H, Tong Z, Ye Q, Sun T, Hong Z, Zhang L, Bortnick A, Cho S, Beuzer P, Axelrod J, Hu Q, Wang M, Evans SM, Murre C, Lu LF, Sun S, Corbett KD, Cang H (2019) Molecular organization of mammalian meiotic chromosome axis revealed by expansion STORM microscopy. Proc Natl Acad Sci USA 116:18423-18428

Zhang S, Zhu M, Shang Y, Wang J, Dawadundup ZL, Zhang J, Chu C, Qi Z (2019) Physical organization of repetitive sequences and chromosome diversity of barley revealed by fluorescence in situ hybridization (FISH). Genome 62:329339

Zhao Y, Bucur O, Irshad H, Chen F, Weins A, Stancu AL, Oh EY, DiStasio M, Torous V, Glass B, Stillman IE, Schnitt SJ, Beck AH, Boyden ES (2017) Nanoscale imaging of clinical specimens using pathology-optimized expansion microscopy. Nat Biotechnol 35:757-764

Zwettler FU, Reinhard S, Gambarotto D, Bell TDM, Hamel V, Guichard P, Sauer M (2020a) Molecular resolution imaging by post-labeling expansion single-molecule localization microscopy (Ex-SMLM). Nat Commun 11:3388

Zwettler FU, Spindler MC, Reinhard S, Klein T, Kurz A, Benavente R, Sauer M (2020b) Tracking down the molecular architecture of the synaptonemal complex by expansion microscopy. Nat Commun 11:3222

Publisher's note Springer Nature remains neutral with regard to jurisdictional claims in published maps and institutional affiliations. 